\title{
Specific structural features of syndecans and heparan sulfate chains are needed for cell signaling
}

C.C. Lopes,

C.P. Dietrich and H.B. Nader
Departamento de Bioquímica, Escola Paulista de Medicina,

Universidade Federal de São Paulo, São Paulo, SP, Brasil

\section{Correspondence}

C.C. Lopes

Departamento de Bioquímica

EPM, UNIFESP

Rua 3 de Maio, 100

04044-020 São Paulo, SP

Brasil

Fax: +55-11-5573-6407

E-mail: lopesazevedo.bioq@epm.br

Research supported by CAPES, CNPq, and FAPESP.

Received June 30, 2005

Accepted November 8, 2005

A...............,

\section{Abstract}

The syndecans, heparan sulfate proteoglycans, are abundant molecules associated with the cell surface and extracellular matrix and consist of a protein core to which heparan sulfate chains are covalently attached. Each of the syndecan core proteins has a short cytoplasmic domain that binds cytosolic regulatory factors. The syndecans also contain highly conserved transmembrane domains and extracellular domains for which important activities are becoming known. These protein domains locate the syndecan on cell surface sites during development and tumor formation where they interact with other receptors to regulate signaling and cytoskeletal organization. The functions of cell surface heparan sulfate proteoglycan have been centered on the role of heparan sulfate chains, located on the outer side of the cell surface, in the binding of a wide array of ligands, including extracellular matrix proteins and soluble growth factors. More recently, the core proteins of the syndecan family transmembrane proteoglycans have also been shown to be involved in cell signaling through interaction with integrins and tyrosine kinase receptors.
Key words

- Syndecans

- Heparan sulfate proteoglycans

- Protein kinase C

- Growth factors

- Cell signaling

\section{Introduction}

Proteoglycans are molecules composed of a protein core to which glycosaminoglycan chains are covalently attached. The syndecans consist of a family of highly conserved type I transmembrane heparan sulfate proteoglycans that are expressed in a developmental and cell type-specific pattern (14). The amino-terminal sequence of the core protein is followed by an ectodomain containing Ser-Gly consensus sequences for glycosaminoglycan attachment, a single highly conserved transmembrane domain and a short highly conserved cytoplasmic domain. All syndecan proteins carry heparan sulfate chains, and some core proteins can be additionally substituted with chondroitin sulfate chains (5). The expression of the syndecans can be altered under certain pathophysiological conditions, including the processes of tumor onset, progression and metastasis $(6,7)$. Many reports have suggested that heparan sulfate proteoglycans play a role in growth control, cell spreading, cellular recognition, cellular adhesion, and signaling, possibly as co-receptors with integrins and cell-cell adhesion molecules, including fibronectin, vi- 
tronectin, laminins, and the fibrillar collagens (1-3,8-12). In addition, several papers have described high affinity association of heparin-like molecules with growth factors, implying that the effects of heparan sulfate on cell growth are likely to be mediated by growth factors (13-18). This review focuses on the possible roles of syndecan core proteins and heparan sulfate chains in cell signaling.

\section{Structure of syndecans}

The syndecan core proteins consist of a family of four distinct genes in vertebrates. The data regarding the sequence of Drosophila syndecan as well as chromosomal locations and exon organization, suggest that the gene family arose by gene duplication and divergent evolution from a single ancestral gene, and that syndecan- 1 and -3 and syndecan-2 and -4 represent subfamilies. Each gene product is a single type I membrane-spanning protein with an apparently extended extracellular domain of varying size that contains covalently attached heparan sulfate chains distal from the plasma membrane (3) (Figure 1).

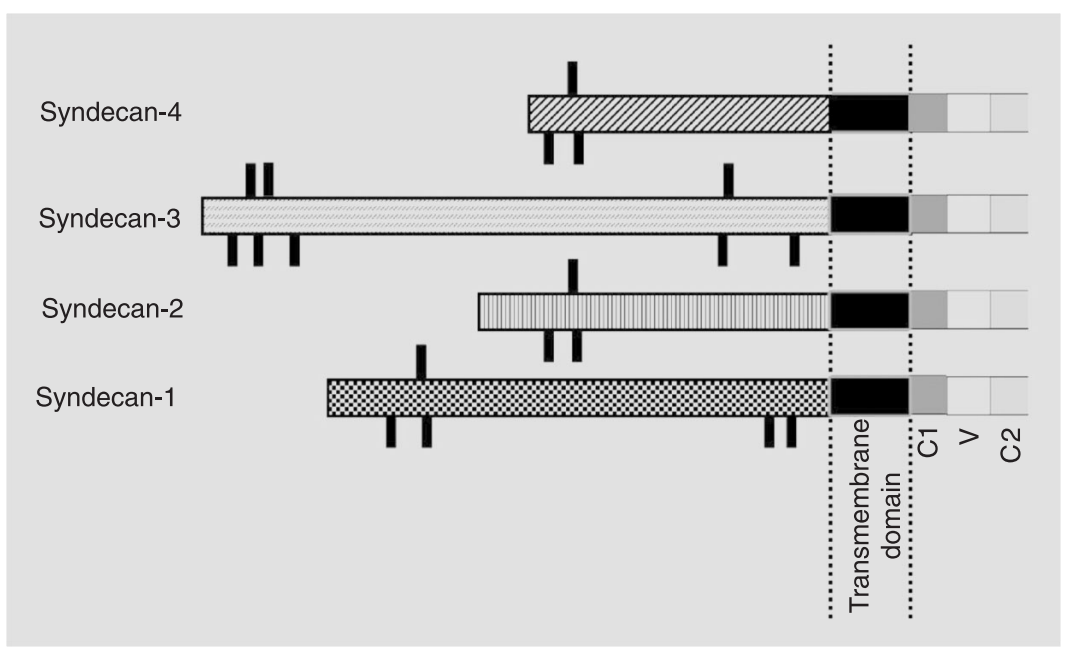

Figure 1. Schematic representation of mammalian syndecans. The proteins are aligned at the signal peptides and the transmembrane domains, leaving gaps in the ectodomains. The glycosaminoglycan attachment sites are indicated as black lines. Syndecans contain a conserved transmembrane domain and a small cytoplasmic domain consisting of one variable $(\mathrm{V})$ and two conserved $(\mathrm{C} 1$ and $\mathrm{C} 2)$ regions.
The syndecans are membrane proteins that do not contain cysteine residues and show few but important structural similarities. Structural variabilities are mainly found in the extracellular domains (ectodomains), that are among the most rapidly diverging vertebrate proteins with the exception of their regions for GAG attachment, cell interaction, proteolytic cleavage site, and oligomerization. These domains contain sequences of Ser-Gly-X-Gly preceded by an acidic amino acid, being consensus sequences for the glycosylation of heparan sulfate chains. Also, protease cleavage sites composed of basic amino acids are found close to the transmembrane portion of the protein core and are related to shedding of the proteoglycan from the cell surface (Figure 1). The transmembrane domains are relatively stable evolutionarily, since only a few amino acids differ among the vertebrate sequences. These domains contain regions for interactions with other membrane proteins and for localization to distinct membrane compartments.

The cytoplasmic domains contain two invariant regions, a membrane proximal common region $(\mathrm{C} 1)$ containing a serine and a tyrosine and a $\mathrm{C}$-terminal common region (C2), separated by a region (V) of variable length and composition (Figures 1 and 2). The $\mathrm{C} 2$ region shows an EFYA sequence at the C-terminus that can bind to the PDZ domain present in specific proteins. PDZ domains, named for PSD-95, Discs-large, and Zonula occludens-1 proteins, bind specific C-terminal sequences and organize and assemble protein complexes on the inner surface of the plasma membrane and are thought to link membrane components to the underlying actin-containing cytoskeleton (Figure 2). The variable (V) region is distinct for each of the 4 family members, but its syndecan specific identity is conserved across species (3). The function of this domain is largely unknown except for syndecan4 , where it is responsible for the assembly of 
syndecan-4 tetramers with phosphatidylinositol 4,5-bisphosphate $\left(\mathrm{PIP}_{2}\right)$ and activated protein kinase $\mathrm{C}-\alpha(\mathrm{PKC}-\alpha)$ in focal adhesions $(19,20)$.

Dramatic changes in syndecan expression occur during development and are associated with morphological transitions, cell differentiation, or changes in tissue organization (21).

\section{Syndecans in cell adhesion}

Intact proteoglycan is mandatory for cell adhesion and migration since these processes require both heparan sulfate-ligand binding and interactions of the core protein with cytoskeletal and/or signaling molecules. The cytoplasmic domain of syndecan interacts with a number of signaling and structural proteins, and both extracellular and cytoplasmic domains are necessary for the activation of transmembrane receptors. All mammalian syndecan cytoplasmic domains contain a conserved EFYA sequence at the Cterminus, which can bind PDZ domain-containing scaffold proteins (CASK and syntenin) and coordinate clustering of receptors and connection to the actin cytoskeleton (Figure 2). As already mentioned, proteins bearing the PDZ domain are implicated in organizing signaling complexes at specific sites of the membrane, such as cell junctions (20, 22).

In vitro studies indicate that all syndecans can interact with CASK, and may form similar complexes in different contexts. CASK forms a ternary complex with syndecan-2 and protein 4.1 , possibly linking the extracellular membrane and the cytoskeleton (23). Syndecan-2 and CASK co-localize in rat brain neuronal synapses, suggesting involvement in signaling at synaptic junctions (24). This complex also seems to be crucial for maturation of dendritic spines of rat hippocampal neurons (25).

The involvement of proteoglycans in cytoskeletal organization has been proposed in other studies $(19,26)$. Cell spreading is promoted by over-expression of syndecan- 1 that transiently co-localizes with microfilaments. Thrombospondin-1 functions as a cell adhesion molecule and also regulates cell adhesion to other matrix components. Syndecan1, together with F-actin and fascin, is involved in signal transduction of cells spreading on thrombospondin-1 (27). Syndecan-2 is found on the actin-rich dendritic spines and may be responsible for the morphological maturation of spines from long thin protrusions to stubby headed shapes (28). Syndecan-3 in Schwann cells mediates cell adhesion and spreading on a novel heparan sulfate binding site located at the $\mathrm{N}$-terminal

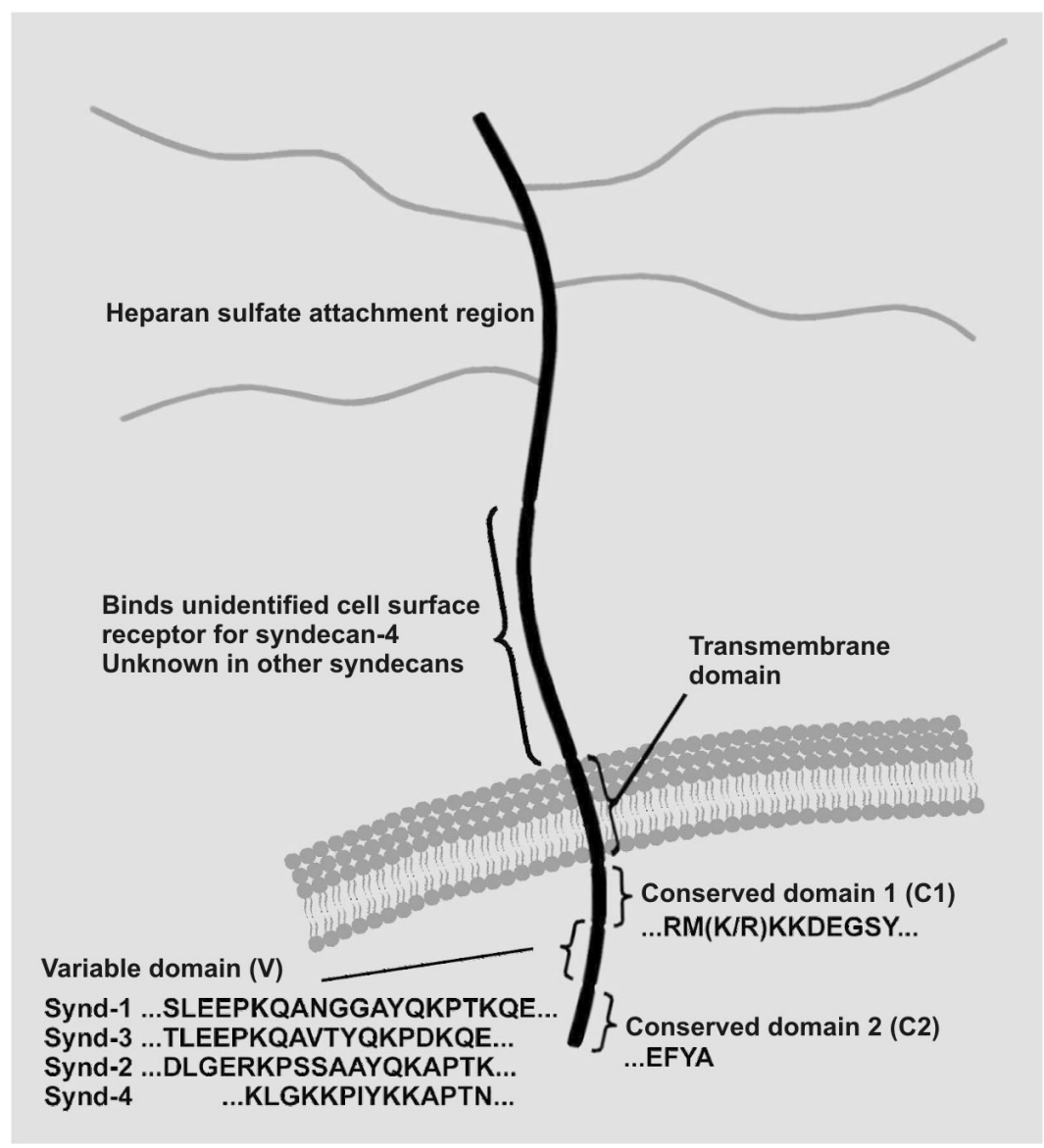

Figure 2. Syndecan core protein structure. The extracellular domains of the four vertebrate syndecans contain several sites for heparan sulfate chain attachment. The transmembrane domain is highly conserved among the family members. The cytoplasmic domains contain regions ( $\mathrm{C} 1$ and $\mathrm{C} 2)$ that are exactly conserved in each of the four syndecans (the exception being a conservative substitution of arginine for lysine in syndecan-2). These flank a central variable $(V)$ region that is distinct for each family member. Synd = syndecan. 
domain of type $\mathrm{V}$ collagen ( $\alpha 4$ chain). This process leads to actin assembly, tyrosine phosphorylation, as well as activation of the Erk1/Erk2 protein kinases (29).

The best evidence for a specific role of syndecans in cell adhesion comes from studies of focal adhesion formation. Focal adhesions are specialized zones of tight cellmatrix interaction located at the termini of actin stress fibers, and are important signaling centers, where integrins and heparan sulfate proteoglycans cooperate to connect extracellular matrix and cytoskeleton $(30,31)$ (Figure 3).

Syndecan-4 is a peculiar transmembrane heparan sulfate proteoglycan present together with integrins in focal adhesion on different substrates (fibronectin, laminin, vitronectin, or type I collagen) of a number of cell types. The syndecan-4 $\mathrm{C} 1$ and $\mathrm{V}$ regions bind syndesmos, a paxillin-binding protein that

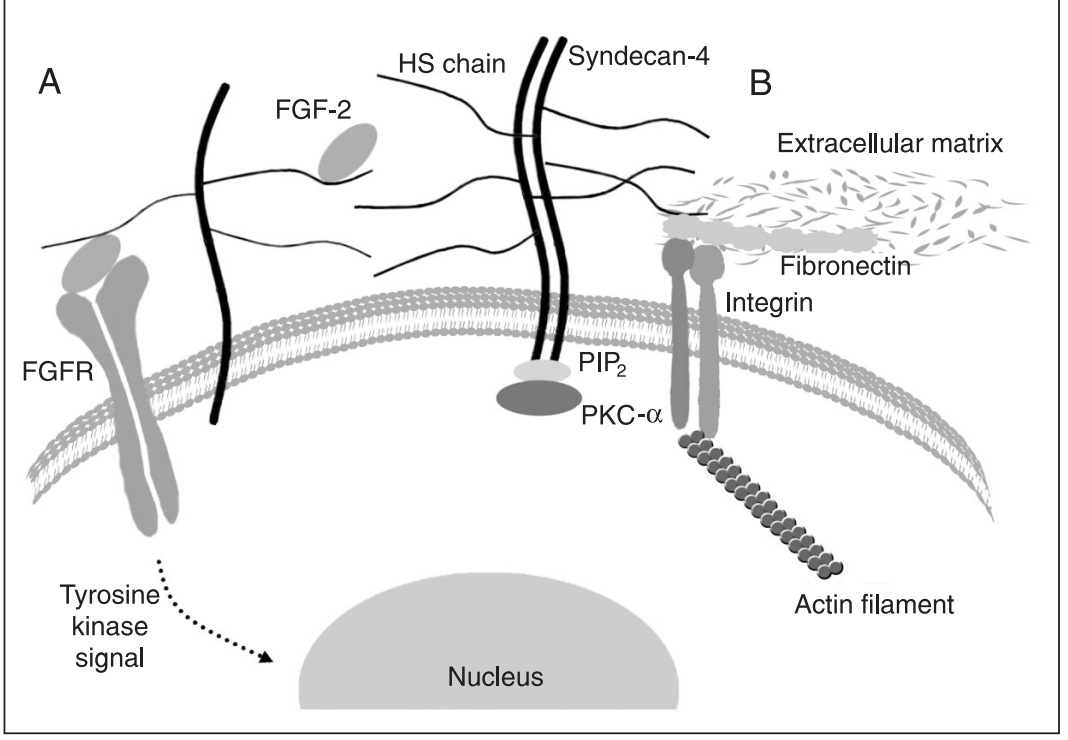

Figure 3. Some of the activities involving syndecans. Syndecans modulate growth factor signaling (A) and participate in cell adhesion (B). A, Binding to syndecans can regulate growth factor interactions with tyrosine kinase receptors. The model shows heparan sulfate (HS) chains mediating dimerization of fibroblast growth factor (FGF)-2 and subsequently FGF receptors (FGFR), promoting the formation of a signaling complex and tyrosine kinase signal transduction. Multiple syndecans were shown to support FGF biological activities. $B$, Focal adhesion is a specialized membrane complex in which integrins and syndecan cooperate to connect extracellular matrix and cytoskeleton. The syndecan involved appears to be exclusively syndecan-4. PIP $2=$ phosphatidylinositol 4,5-bisphosphate; PKC- $\alpha$ $=$ protein kinase $\mathrm{C}-\alpha$. may serve to link syndecan-4 to the cytoskeleton. $\alpha$-Actinin has also been recently shown to bind the $\mathrm{V}$ region of syndecan-4 (19).

Primary fibroblasts attach and spread following integrin ligation, but do not form focal adhesions in the absence of a heparinbiding domain of fibronectin, implying requirement of heparan sulfate proteoglycan $(3,19,32)$. Recruitment of syndecan-4 into focal adhesions may be dependent on activation of PKC. A connection was established between the $\mathrm{V}$ region of syndecan-4 and PKC- $\alpha$ localization and activation, consistent with a role for this kinase in fibroblast focal adhesion assembly $(19,32)$.

\section{Syndecan-4 and PKC activation}

A family of serine/threonine kinases collectively known as PKC are involved in the regulation of a variety of cell functions including proliferation, gene expression, cell cycle, differentiation, cytoskeletal organization, cell migration, cell adhesion, and apoptosis. In the syndecan family, syndecan-4 is the one related to PKC activation. Syndecan4 cytoplasmic domain interacts with PKC- $\alpha$ (19,32-34) and activates its kinase activity in the presence of $\mathrm{PIP}_{2}$ in vitro $(21,34)$. In vivo, syndecan-4 also interacts with PKC- $\alpha$ and this interaction regulates localization of PKC- $\alpha$ into the cytoskeleton, resulting in a sustained PKC activity (34).

Oligomerization of syndecan- 4 cytoplasmic domains results from the interaction with $\mathrm{PIP}_{2}$ (32). Studies have shown that the dimer formed by the syndecan- $4 \mathrm{~V}$ region can bind PIP $_{2}$ and is stabilized by the interaction (35). However, the number of dimers and PIP $_{2}$ molecules involved in a single signaling complex remains to be determined. Nevertheless, it is clear that oligomerization of the syndecan-4 proteoglycan not only drives focal adhesion formation but also provides a platform for the binding and activation of PKC- $\alpha$ (Figure 3). 
Work with fusion proteins and synthetic peptides shows that oligomerized, but not monomeric, cytoplasmic domains of syndecan- 4 can bind PKC- $\alpha$ directly through the PKC- $\alpha$ catalytic domain, which in turns results in direct activation of PKC- $\alpha$ in the absence of other mediators and superactivation in their presence. The combination of the syndecan-4 cytoplasmic domain, $\mathrm{PIP}_{2}$ and PKC- $\alpha$ yields a potent signaling complex, in which levels of PKC- $\alpha$ activity are as high as can be achieved using conventional activators. The signaling activity of the cytoplasmic tail of syndecan- 4 seems to be supported by $\mathrm{PIP}_{2}$ that serves as a binding interface, an essential co-factor for PKC- $\alpha$ activation and a facilitator for the multimerization of the tail (33-36). These properties are regulated by the phosphorylation of a specific serine residue located close to the $\mathrm{N}$-terminus of the $\mathrm{PIP}_{2}$ binding motif (35). $\mathrm{PKC}-\delta$, rather than PKC- $\alpha$ that associates with the domain in focal adhesions, appears to be responsible for the phosphorylation event (36). Once phosphorylated, the affinity of the cytoplasmic tail for $\mathrm{PIP}_{2}$ and its capacity to oligomerize and activate PKC- $\alpha$ in the presence of $\mathrm{PIP}_{2}$ are sharply reduced (33). The association of syndecan- 4 with PKC- $\alpha$ puts the proteoglycan in a category similar to other transmembrane receptors, such as integrin, which do not possess intrinsic catalytic activity, but are associated with kinases or phosphatases.

\section{Syndecans and growth factor signaling}

Growth factors are polypeptide molecules that elicit several responses in target cells. These responses include proliferation, differentiation, survival, and apoptosis of the cells. A large number of growth factors are known to bind syndecans through their heparan sulfate chains (37), including members of the fibroblast growth factor (FGF) family. FGF binds to the extracellular domain of its receptor, which is a transmembrane molecule with protein-tyrosine kinase activity in the cytoplasmic region. After growth factor binding, the dimerization of the receptor occurs, followed by autophosphorylation of the intracellular domain of the receptor. This process will initiate a signal transduction cascade which will generate signals that reach the nucleus, activating the expression of specific genes and leading to the cellular response (Figure 3).

As co-receptors, syndecans increase the local concentrations of growth factors and promote receptor dimerization (3). Heparan sulfate proteoglycan mediates the binding of FGF to its high affinity receptor, which has tyrosine kinase activity (13). Syndecan-4 over-expression enhances FGF-2 signaling, and chimeric receptors composed of the syndecan-4 cytoplasmic domain and heparan sulfate-substituted extracellular domains enhance the cellular response to FGF, but this is not the case for chimeras lacking the cytoplasmic domain $(38,39)$, a fact implying the importance of syndecan-4 cytoplasmic domain-mediated PKC activation in FGF signaling. In contrast, there is no clear evidence for syndecan-2 regulation of FGF signaling in fibroblasts. However, syndecan-2 in activated human macrophages can selectively bind FGF-2, vascular endothelial growth factor and heparin-binding endothelial growth factor. These results imply a key role for syndecan-2 in the delivery of sequestered growth factors by inflammatory macrophages for productive binding to their appropriate target cells in vivo (37).

In addition to FGF, syndecans- 2 and -4 participate in the regulation of other growth factors. It has been suggested that syndecan4 regulates protein levels of the endothelial growth factor receptor (erb-B2 and -B3) in a colon cancer cell line (40). Functional interactions between syndecan-2 and GM-CSF in osteoblasts have been suggested; perhaps syndecan-2 also plays a role as a co-receptor for this cytokine (41). Moreover, syndecan- 
2 can bind and regulate the signaling of tumor growth factor- $\beta$, and the binding is through the ectodomain of the core protein, with the heparan sulfate chains having only a minor role. The binding is independent of the cytoplasmic domain (42).

Syndecan-1 is also implicated in growth factor signaling. Hepatocyte growth factor binding to syndecan-1 promotes activation of PI3-kinase and mitogen-activated protein kinase pathways. Soluble syndecan-1 appears to act as a carrier for hepatocyte growth factor in vivo and has been implicated in the pathology of myeloma by modulating cytokine activity within the bone marrow (43). Syndecan-1, over-expressed on 3T3 cells has been shown to inhibit FGF-2 signaling. Syndecan-1 induction in S115 mammary carcinoma cells can prevent the androgeninduced transformation of these cells and this can be accomplished by adding the soluble ectodomain of syndecan-1 from normal NMuMG mammary epithelial cells, suggesting disruption of an autocrine signaling pathway that may involve FGF-2, FGF-8 or other heparin-binding growth factors (44).

Members of the FGF family mediate, at least in part, the outgrowth of the mesoderm of the developing limb bud in response to the apical ectodermal ridge. It has been shown that syndecan-3 plays an essential role in this process, mediating the interaction of the FGFs produced by the apical ectodermal ridge with the underlying mesoderm of the limb bud. In addition, syndecan-3 also is a cell-surface receptor for heparin-binding growth factor-associated molecule (HBGAM) and is implicated in the activity of this molecule on neurite outgrowth. On the other hand, it should be mentioned that HBGAM interacts with the heparan sulfate chains of syndecan-3. Syndecan-3 is a receptor for HB-GAM which promotes neurite outgrowth. This requires c-Src and Fyn tyrosine kinase activity, and involves cortactin and tubulin. All of these molecules coimmunoprecipitate with the cytoplasmic do- main of syndecan-3, suggesting the assembly or activation of a tyrosine kinase-containing cytoplasmic complex $(38,45,46)$.

Furthermore, syndecans are shed from cell surfaces via cleavage of their ectodomain. Syndecan shedding releases the ectodomain as a soluble regulatory macromolecule. Syndecan- 1 and -4 ectodomains in wound fluids bind to growth factors, proteases and protease inhibitors. FGF-2, which is dependent on heparan sulfate for its activity, is inhibited by binding to the glycosaminoglycan of the soluble syndecan- 1 ectodomain. Cleavage of these heparan sulfate chains by physiological heparinase produces soluble fragments that greatly enhance FGF-2 activity. Thus, it is not known under what circumstances a proteoglycan may be inhibitory or stimulatory to growth factor signaling (22, 47).

\section{Heparan sulfate chains and cell signaling}

Ligand binding was once thought to be a relatively nonspecific ionic interaction between negatively charged heparin and heparan sulfate and positively charged domains of proteins. However, many ligands require specific sequences of well-defined length and structure. Nowadays, several lines of evidence suggest that the structural peculiarities may be related to heparan sulfate functions. As already mentioned, heparan sulfate proteoglycans are present on the cell surface of mammalian cells in culture and in most vertebrate and invertebrate tissues ( 1 , $2,9)$.

The advance of the chemistry and biology and the increasing knowledge of the physicochemical properties of heparin and heparan sulfate have permitted us to clearly distinguish between these two types of glycosaminoglycans. This identification is based on a number of criteria, including structural characteristics, cellular localization and anticoagulant activity (Table 1). Heparan sul- 
fates are composed of alternating units of $\alpha$ D-glucosamine and uronic acid ( $(B-D$-glucuronic or $\alpha$-L-iduronic acid) and the hexosamine is either $N$-acetylated or $N$-sulfated and/or 6- $O$-sulfated. Uronic acid can also be 2- $O$ sulfated. The heparan sulfates exhibit a peculiar structural variability (degree of $N$ sulfation/ $\mathrm{N}$-acetylation and 6-O-sulfation as well as the ratio of glucuronic acid and iduronic acid) according to the tissue and species of origin. Sequence analyses of heparan sulfates have shown that all of them are co-polymers consisting of several oligosaccharide regions with distinct disaccharide composition (Figure 4). These differences could explain, for example, the preferential binding of growth factors to some cells but not to others.

The action of heparan sulfate chains as positive modulators of cell proliferation is due to the capacity of the compound to bind and act as a co-receptor for growth factors. They potentiate the action of low concentrations of growth factor by enhancing the formation of growth factor-receptor complexes.

Sulfated oligosaccharides obtained from heparin and heparan sulfate potentiate the mitogenic activity of FGF-1 on 3T3 fibroblasts. Although heparan sulfates extracted from several mammalian tissues potentiated the mitogenic activity of FGF-1, in general, they were less efficient than heparin on a mass basis. On the other hand, an oligosaccharide obtained from heparan sulfate after digestion with heparitinase I is more active than the intact molecule, reaching a potentiating effect equivalent to that of heparin, whereas an $\mathrm{N}$-acetylated oligosaccharide isolated after nitrous acid degradation is inactive. The results indicate that the mitogenic activity of FGF-1 is primarily potentiated through the interaction of more sulfated regions of the heparan sulfate chains as well as the presence of conformationally flexible iduronic acid residues. Curiously, heparan sulfate prepared from 3T3-conditioned culture medium proved to be more efficient than heparin both on a mass and molar basis. These results suggest that there is specificity for this interaction, favoring the structural specificity of this process (14). In this regard, it has been shown that $N$-sulfate groups and saccharides containing iduronic acid 2$O$-sulfate are essential for the high affinity

Table 1. Comparison of heparin and heparan sulfate.

\begin{tabular}{lcc}
\hline Parameter & Heparin & Heparan sulfate \\
\hline Potassium acetate $\left(2 \mathrm{M}, \mathrm{pH} 5.7,4^{\circ} \mathrm{C}\right)$ & Insoluble & Soluble \\
Band formation in electrofocusing & + & - \\
Electrophoretic mobility (agarose) & & 0.7 \\
$\quad$ Barbital & 1.0 & 1.0 \\
Diaminopropane & 1.0 & 2.0 \\
Barium - diaminopropane & 1.0 & 525 \\
Metachromatic activity (nm) & 505 & $0.8-1.8^{\mathrm{b}}$ \\
Sulfate/hexosamine ratios & $2.4-2.8^{\mathrm{a}}$ & $<5^{\mathrm{c}}$ \\
Anticoagulant activity (USP/mg) & $60-320$ & \\
Degradation & & + \\
Heparinase & + & + \\
Heparitinase I & - & + \\
Heparitinase II & + & + \\
Cellular localization & & + \\
Granules & + & + \\
Cell surface & - & + \\
Extracellular matrix & - & less than $5 \%$ \\
Binding & + & + \\
Antithrombin & + & + \\
Growth factors & + & \\
\hline
\end{tabular}

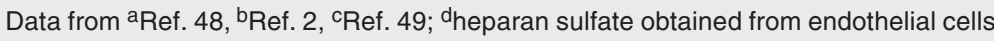
in culture is degraded by heparinase to the extent of $20 \%$ (Ref. 50 ).

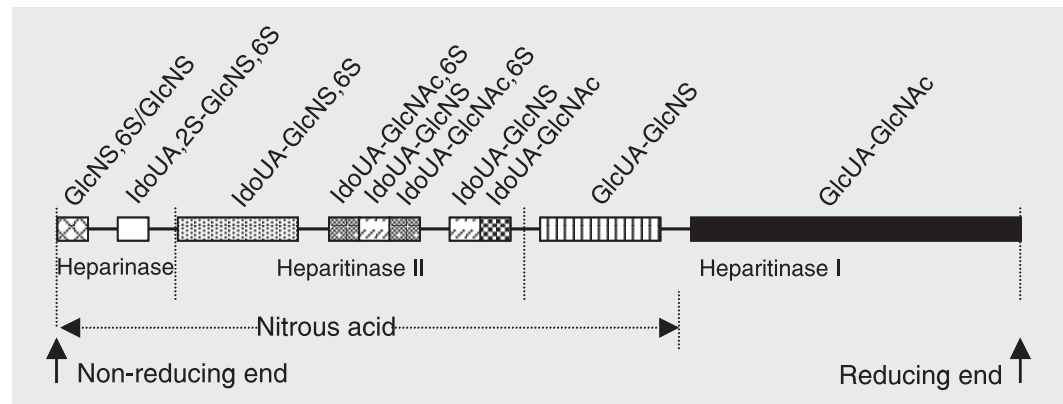

Figure 4. Proposed general structure of heparan sulfate and sites of cleavage of heparitinase I, heparitinase II, heparinase, and nitrous acid (for details, see Ref. 15). GIcNAc $=2$ acetamido $\alpha$-D-glucopyranose; GlcNAc,6S = 2-acetamido $\alpha$-D-glucopyranose-6-O-sulfate; GlcNS $=2$ - $N$-sulfoamino $\alpha$-D-glucopyranose; GlcNS, $6 S=2$ - $N$-sulfoamino $\alpha$-D-glucopyranose-6-O-sulfate; GlcUA = B-D-glucopyranosyl uronic acid; IdoUA $=\alpha$-L-idopyranosyl uronic acid; IdoUA,2S $=\alpha$-L-idopyranosyl uronic acid 2-O-sulfate. 
binding of human skin fibroblast heparan sulfate to FGF-2 and the sequence of an oligosaccharide with similar affinity for the growth factor as the parent molecule has been determined (51).

It has been demonstrated that $N$-sulfate groups are most important, followed by 2-Oand 6-O-sulfate groups, for binding to neuregulin-1 (NRG-1) and blocking erbB receptor phosphorylation. Inhibition of sulfation through the use of chlorate or selective blockade of $N$-sulfation with a siRNA directed against the enzyme that promotes $N$ sulfation resulted in decreased NRG-1-induced erbB receptor activation. This specificity provides a means by which tissues can localize and potentiate NRG-1 signaling through modifications in glycosaminoglycan composition (52).

Recently, it has been shown that the binding of endostatin to endothelial heparan sulfate shows a differential requirement for specific sulfate groups. The results indicate that 6-O-sulfates play a dominant role in selectivity and $2-O$-sulfates are not strictly essential (53).

Using chlorate-treated rat mammary fibroblasts, FGF-2 and heparin-derived oligosaccharides, dependence on the size of the fragment was demonstrated for the stimulus of DNA synthesis and phosphorylation of p42/44 mitogen-activated protein kinase. However, tetra- to octa-saccharides were less potent in proliferation assays than deca- and longer oligosaccharides. Furthermore, there was no correlation between the binding parameters and the potency of the oligosaccharides in DNA synthesis assays (54).

The phorbol ester, phorbol 12-myristate 13-acetate (PMA), was first known for its tumor-promoting activity, and only later was it recognized as a strong and specific activator of PKC, mimicking the natural modulator of this enzyme, i.e., diacylglycerol. We have reported that PMA specifically stimulates the synthesis of heparan sulfate proteoglycan from endothelial cells, an effect that is likely to be mediated by PKC activation. Staurosporine, a potent kinase inhibitor, abolishes the effect of PMA, whereas activation of the cAMP/PKA pathway by both forskolin and/or 8-BrcAMP is not effective in triggering stimulation of heparan sulfate proteoglycan (syndecan-4) synthesis. Furthermore, 18-h PMA pre-treatment, that presumably down-regulates PKC, renders endothelial cells resistant to a second PMA treatment. The most remarkable aspect of these results, however, was the correlation between stimulation of heparan sulfate proteoglycan synthesis and the blockade of $\mathrm{G}_{1}-\mathrm{S}$ phase transition, both triggered by PMA $(55,56)$. The combination of orto-phenyl- $\beta$-D-xylosides with PMA produced some cumulative effect. PMA stimulates the synthesis of heparan sulfate mainly at the $\mathrm{G}_{1}$ phase, whereas the greatest enhancement of synthesis produced by the xylosides is in the $\mathrm{S}$ phase of the endothelial cell cycle (57).

\section{Future directions}

The post-genomic research era has started through proteomic approaches that include the post-translational modifications of proteins. One important point is that the diversity of the proteome may be determined by sugars and phosphorylation. In all studies referred to here, the data clearly indicate that the glycosylation of specific proteins leading to the biosynthesis of syndecans plays major roles in development, differentiation and oncogenesis. The genes involved in the biosynthetic pathway of the heparan sulfate chains are expressed in a manner specific for organs, tissues and cells. Therefore, their activities may differ completely depending on the site of their expression, as well as in pathological conditions such as cancer. Several intriguing biological functions have been demonstrated for heparan sulfate by the analysis of mutants of model organisms and manipulation of genes encoding biosynthetic enzymes. For example, in Drosophila, mu- 
tations have been identified in the homologue of UDP-D-glucose dehydrogenase, which produces UDP-glucuronic acid (sugarless, $s g l)(58)$ and in the $N$-deacetylase $/ N$ sulfotransferase homologue (sulfateless, $s f l$ ) (59). In either $s g l$ or $s f l$ mutant embryos, Wingless (Wg), a member of the Wnt family, FGF and Hedgehog (Hh) signaling pathways are impaired. The phenotype associated with these mutations is severe and corresponds to complete loss of activity of these pathways, indicating that the heparan sulfates are essential for signaling of growth factors and morphogens. Mouse embryos homozygous for a mutation in the gene coding for heparan sulfate 2- $O$-sulfotransferase, also present multiple developmental abnormalities including lack of kidney induction. The abnormalities are probably due to compromised activities of heparan sulfate and growth factors in organogenesis (60).

As already noted, heparan sulfate chains are consistently found in members of the family of transmembrane proteoglycans, known as syndecans. Syndecans participate in a variety of physiological processes. They appear at the early stages of development, and the glycosaminoglycan chains vary according to the tissue and species of origin.
Structural modifications are found in diseases, especially in neoplastic transformation. Recent advances in syndecan research suggest that the core proteins of these proteoglycans are involved in many signaling functions that translate information to the cytosol from binding events of their heparan sulfate chains during developmental processes. The mechanism by which syndecans can act as co-receptors with integrins remains unknown, but recent studies point to the syndecan extracellular protein domains, that appear to complement the binding interactions of their attached heparan sulfate chains. Syndecan-4 can also interact with PKC and stimulate its activity. The syndecans modulate the actions of a wide variety of heparan sulfate-binding proteins.

Understanding the mechanisms by which the syndecans participate in signal transduction pathways and questions addressing the manner in which the enzymes related to the biosynthesis of these compounds are located in the cell or in the Golgi complex, what kind of cluster they form, and how they actually synthesize the sugar chain, are the new frontier for this area, and may lead to novel therapeutic strategies for the control of tumor cell invasion and metastasis.

\section{References}

1. Dietrich CP, Nader HB \& Straus AH (1983). Structural differences of heparan sulfates according to the tissue and species of origin. Biochemical and Biophysical Reserch Communications, 111: 865871.

2. Dietrich CP, Tersariol ILS, Toma L et al. (1998). Structure of heparan sulfate: identification of variable and constant oligosaccharide domains in eight heparan sulfates of different origins. Cellular and Molecular Biology, 44: 417-429.

3. Bernfield M, Gotte M, Park PW et al. (1999). Functions of cell surface heparan sulfate proteoglycans. Annual Review of Biochemistry, 68: 729-777.

4. Kim CW, Goldberger OA, Gallo RI et al. (1994). Members of the syndecan family of heparan sulfate proteoglycans are expressed in distinct cell-, tissue-, and development-specific patterns. Molecular Biology of the Cell, 5: 797-805.

5. Ueno M, Yamada S, Zako M et al. (2001). Structural characteriza- tion of heparan sulfate and chondroitin sulfate of syndecan-1 purified from normal murine mammary gland epithelial cells. Common phosphorylation of xylose and differential sulfation of galactose in the protein linkage region tetrasaccharide sequence. Journal of Biological Chemistry, 276: 29134-29140.

6. Sanderson RD (2001). Heparan sulfate proteoglycans in invasion and metastasis. Seminars in Cell and Developmental Biology, 12: 89-98.

7. Sasisekharan R, Shriver Z, Venkataraman G et al. (2002). Roles of heparan sulphate glycosaminoglycans in cancer. Nature Reviews. Cancer, 2: 521-528.

8. Dietrich CP, Sampaio LO, Toledo OM et al. (1977). Cell recognition and adhesiveness: a possible biological role for the sulfated mucopolysaccharides. Biochemical and Biophysical Research Communications, 75: 329-336.

9. Dietrich CP (1984). A model for cell-cell recognition and control of 
cell growth mediated by sulfated glycosaminoglycans. Brazilian Journal of Medical and Biological Research, 17: 5-15.

10. Fransson LA (1989). Heparan sulphate proteoglycans: structure and properties. In: Lane DA \& Lindhal U (Editors), Heparin. Chemical and Biological Properties and Clinical Applications. Edward Arnold, London, UK.

11. Tumova S, Woods A \& Couchman JR (2000). Heparan sulfate proteoglycans on the cell surface: versatile coordinators of cellular functions. International Journal of Biochemistry and Cell Biology, 32: 268-288.

12. Thodeti CK, Albrechtsen R, Grauslund M et al. (2003). ADAM12/ syndecan-4 signaling promotes beta(1) integrin-dependent cell spreading through protein kinase $\mathrm{C}$ alpha and RhoA. Journal of Biological Chemistry, 278: 9576-9584.

13. Yayon A, Klagsbrun M, Esko JD et al. (1991). Cell surface heparinlike molecules are required for binding of basic fibroblast growth factor to its high affinity receptor. Cell, 64: 841-848.

14. Gambarini AG, Miyamoto CA, Lima GA et al. (1993). Mitogenic activity of acidic fibroblast growth factor is enhanced by highly sulfated oligosaccharides derived from heparin and heparan sulfate. Molecular and Cellular Biochemistry, 124: 121-129.

15. Schlessinger J, Lax I \& Lemmon M (1995). Regulation of growth factor activation by proteoglycans: what is the role of the low affinity receptors? Cell, 83: 357-360.

16. Porcionatto MA, Nader HB \& Dietrich CP (1999). Heparan sulfate and cell division. Brazilian Journal of Medical and Biological Research, 32: 539-544.

17. Lin X (2004). Functions of heparan sulfate proteoglycans in cell signaling during development. Development, 131: 6009-6021.

18. Presta M, Dell'era P, Mitola S et al. (2005). Fibroblast growth factor/ fibroblast growth factor receptor system in angiogenesis. Cytokine and Growth Factor Reviews, 16: 159-178.

19. Woods A \& Couchman JR (2001). Syndecan-4 and focal adhesion function. Current Opinion in Cell Biology, 13: 578-583.

20. Rapraeger AC \& Otto VL (1998). Molecular interactions of the syndecan core proteins. Current Opinion in Cell Biology, 10: 620628.

21. Beauvais DM \& Rapraeger AC (2004). Syndecans in tumor cell adhesion and signaling. Reproductive Biology and Endocrinology, 2: 1-12.

22. Bass MD \& Humphries MJ (2002). Cytoplasmic interactions of syndecan-4 orchestrate adhesion receptor and growth factor receptor signaling. Biochemical Journal, 368: 1-15.

23. Cohen AR, Wood DF, Marfatia SM et al. (1998). Human CASK/LIN2 binds syndecan-2 and protein 4.1 and localizes to the basolateral membrane of epithelial cells. Journal of Cell Biology, 142: 129-138.

24. Hsueh YP, Yang FC, Kharazia V et al. (1998). Direct interaction of CASK/LIN-2 and syndecan heparan sulfate proteoglycan and their overlapping distribution in neuronal synapses. Journal of Cell Biology, 142: 139-151.

25. Ethell IM \& Yamaguchi $Y$ (1999). Cell surface heparan sulfate proteoglycan syndecan-2 induces the maturation of dendritic spines in rat hippocampal neurons. Journal of Cell Biology, 144: 575-586.

26. Carey DJ (1997). Syndecans: multifunctional cell-surface co-receptors. Biochemical Journal, 327: 1-16.

27. Adams JC, Kureishy N \& Taylor AL (2001). A role for syndecan-1 in coupling fascin spike formation by thrombospondin-1. Journal of Cell Biology, 152: 1169-1182.

28. Halpain S (2000). Actin and the agile spine: how and why do dendritic spines dance? Trends in Neurosciences, 23: 141-146.

29. Erdman R, Stahl RC, Rothblum K et al. (2002). Schwann cell adhe- sion to a novel heparan sulfate binding site in the $\mathrm{N}$-terminal domain of alpha 4 type $\mathrm{V}$ collagen is mediated by syndecan-3. Journal of Biological Chemistry, 277: 7619-7625.

30. Yoneda A \& Couchman JR (2003). Regulation of cytoskeletal organization by syndecan transmembrane proteoglycans. Matrix Biology, 22: 25-33.

31. Baciu PC, Saoncella S, Lee SH et al. (2000). Syndesmos, a protein that interacts with the cytoplasmic domain of syndecan-4, mediates cell spreading and actin cytoskeletal organization. Journal of Cell Science, 113: 315-324.

32. Oh ES \& Couchman JR (2004). Syndecans-2 and -4; close cousins, but not identical twins. Molecular Cell, 17: 181-187.

33. Lim ST, Longley RI, Couchman JR et al. (2003). Direct binding of syndecan-4 cytoplasmic domain to the catalytic domain of protein kinase $\mathrm{C} \alpha(\mathrm{PKC} \alpha)$ increases focal adhesion localization of $\mathrm{PKC} \alpha$. Journal of Biological Chemistry, 278: 13795-13802.

34. Simons M \& Horowitz A (2001). Syndecan-4-mediated signaling. Cell Signal, 13: 855-862.

35. Keum E, Kim Y, Kim J et al. (2004). Syndecan-4 regulates localization, activity and stability of protein kinase C-alpha. Biochemical Journal, 378: 1007-1014.

36. Murakami M, Horowitz A, Tang S et al. (2002). Protein kinase C $(\mathrm{PKC})-\delta$ regulates $\mathrm{PKC}-\alpha$ activity in a syndecan-4-dependent manner. Journal of Biological Chemistry, 277: 20367-20371.

37. Gotte M (2003). Syndecans in inflammation. FASEB Journal, 17: 575-591.

38. Volk R, Schwartz JJ, Li J et al. (1999). The role of syndecan cytoplasmic domain in basic fibroblast growth factor-dependent signal transduction. Journal of Biological Chemistry, 274: 24417-24424.

39. Horowitz A, Tkachenko E \& Simons M (2002). Fibroblast growth factor-specific modulation of cellular response by syndecan-4. Journal of Biological Chemistry, 157: 715-725.

40. Zvibel I, Brill S, Halpern Z et al. (2001). Soluble and matrix-associated heparan sulfate proteoglycans increase expression of erb-B2 and erb-B3 in colon cancer cell lines. International Journal of Cancer, 91: 316-321.

41. Modrowski D, Basle M, Lomri A et al. (2000). Syndecan-2 is involved in the mitogenic activity and signaling of granulocyte-macrophage colony-stimulating factor in osteoblasts. Journal of Biological Chemistry, 275: 9178-9185.

42. Chen L, Klass C \& Woods A (2004). Syndecan-2 regulates transforming growth factor-beta signaling. Journal of Biological Chemistry, 279: 15715-15718.

43. Derksen PW, Keehnen RM, Evers LM et al. (2002). Cell surface proteoglycan syndecan-1 mediates hepatocyte growth factor binding and promotes Met signaling in multiple myeloma. Blood, 99: 1405-1410.

44. Jaakkola $P$ \& Jalkanen $M$ (1999). Transcriptional regulation of syndecan-1 expression by growth factors. Progress in Nucleic Acid Research and Molecular Biology, 63: 109-138.

45. Kosher RA (1998). Syndecan-3 in limb skeletal development. Microscopy Research and Technique, 43: 123-130.

46. Kinnunen T, Raulo E, Nolo R et al. (1996). Neurite outgrowth in brain neurons induced by heparin-binding growth-associated molecule (HB-GAM) depends on the specific interaction of HB-GAM with heparan sulfate at the cell surface. Journal of Biological Chemistry, 271: 2243-2248.

47. Rapraeger AC (2001). Molecular interactions of syndecans during development. Seminars in Cell and Developmental Biology, 12: 107-116.

48. Nader HB \& Dietrich CP (1989). Natural occurrence, and possible 
biological role of heparin. In: Lane DA \& Lindhal U (Editors), Heparin. Chemical and Biological Properties Clinical Applications. Edward Arnold, London, UK.

49. Nader HB, Lopes CC, Rocha HA et al. (2004). Heparins and heparinoids: occurrence, structure and mechanism of antithrombotic and hemorrhagic activities. Current Pharmaceutical Design, 10: 951-966.

50. Nader HB, Dietrich CP, Buonassisi V et al. (1987). Heparin sequences in the heparan sulfate chains of an endothelial cell proteoglycan. Proceedings of the National Academy of Sciences, USA, 84: 3565-3569.

51. Turnbull JE, Fernig DG, Ke Y et al. (1992). Identification of the basic fibroblast growth factor binding sequence in fibroblast heparan sulfate. Journal of Biological Chemistry, 267: 10337-10341.

52. Pankonin MS, Gallagher JT \& Loeb JA (2005). Specific structural features of heparan sulfate proteoglycans potentiate neuregulin-1 signaling. Journal of Biological Chemistry, 280: 383-388.

53. Blackhall FH, Merry CLR, Lyon M et al. (2003). Binding of endostatin to endothelial heparan sulphate shows a differential requirement for specific sulphates. Biochemical Journal, 375: 131-139.

54. Delehedde M, Lyon M, Gallagher JT et al. (2002). Fibroblast growth factor-2 binds to small heparin-derived oligosaccharides and stimulates a sustained phosphorylation of p42/44 mitogen-activated protein kinase and proliferation of rat mammary fibroblasts. Biochemi- cal Journal, 366: 235-244.

55. Porcionatto MA, Pinto CR, Dietrich CP et al. (1994). Heparan sulfate proteoglycan and control of cell proliferation: enhanced synthesis induced by phorbol ester (PMA) during G(1)-phase. Brazilian Journal of Medical and Biological Research, 27: 2185-2190.

56. Porcionatto MA, Moreira CR, Armelin HA et al. (1998). Stimulation of heparan sulfate proteoglycan synthesis and secretion during $\mathrm{G}_{1}$ phase induced by growth factors and PMA. Journal of Cellular Biochemistry, 70: 563-572.

57. Moreira CR, Lopes CC, Cuccovia IM et al. (2004). Heparan sulfate and control of endothelial cell proliferation: increased synthesis during the $S$ phase of the cell cycle and inhibition of thymidine incorporation induced by ortho-nitrophenyl-beta-D-xylose. Biochimica et Biophysica Acta, 1673: 178-185.

58. Binari RC, Staveley BE, Johnson WA et al. (1997). Genetic evidence that heparin-like glycosaminoglycans are involved in wingless signaling. Development, 124: 2623-2632.

59. Lin X \& Perrimon N (1999). Dally cooperates with Drosophila Frizzled 2 to transduce Wingless signaling. Nature, 400: 281-284.

60. Bullock SL, Fletcher JM, Beddington RSP et al. (1998). Renal agenesis in mice homozygous for a gene trap mutation in the gene encoding heparan sulfate 2-sulfotransferase. Genes and Development, 12: 1894-1906. 